\title{
Clinical Analysis of Microscopic Removal of Discal Cyst
}

\author{
Eui Seok Wang ${ }^{1}$, Chul Gab Lee ${ }^{2}$, Seok Won Kim', Yun Sung Kim³, Dong Min Kim \\ Departments of Neurosurgery ${ }^{1}$, Environmental Medicine ${ }^{2}$, Internal Medicine ${ }^{3}$, College of Medicine, \\ Chosun University, Gwangju, Republic of Korea
}

Objective: The purpose of this study was to evaluate the clinical presentation and surgical outcome in patients with symptomatic discal cyst.

Methods: The authors reviewed consequent 9 patients in whom microscopic excision of the discal cyst with or without additional discectomy for discal cyst from 2005 to 2012. Diagnostic imagings including simple radiographs, computed tomography with discogram and magnetic resonance images were performed in each case. The patients were reviewed to evaluate the clinical presentation, surgical outcome and related complications.

Results: In all patients, discal cyst was located in the lumbar region and they presented with back pain and unilateral radiating pain. The preoperative magnetic resonance images (MRI) and computed tomography (CT) scan with discogram showed a connection between the cyst and the involved intervertebral disc. All patients obtained immediate relief of symptoms after microscopic excision of discal cyst. There were no recurrent lesions during follow-up period. The mean preoperative visual analogue scale (VAS) was 7.8 when compared with 2.6 in preoperative assessment. All patients obtained excellent or good outcome according to modified MacNab's criteria.

Conclusion: Discal cysts are rare lesions that can lead to back pain and refractory sciatica. Microscopic excision of the cyst can achieve remarkable improvement of symptoms.

Key Words: Discal cyst $\cdot$ Excision $\cdot$ Microscope

\section{INTRODUCTION}

Discal cysts are referred as intraspinal extraepidural cysts with a distinct connection to the corresponding intervertebral discs ${ }^{4}$. They are rare and cause indistinguishable symptoms from lumbar disc herniation. Moreover, the clinical manifestations and pathological features of discal cyst have not yet been completely understood. As far as the authors' knowledge, the surgical results of microscopic excision of discal cyst have not been reported except for some case reports ${ }^{2,3,5}$. The authors report the surgical outcome of nine patients with symptomatic radiculopathy caused by discal cysts to discuss the

\footnotetext{
- Received: March 15, 2013 - Revised: June 25, 2013

- Accepted: June 27, 2013

Corresponding Author: Seok Won Kim, MD, PhD

Department of Neurosurgery, School of Medicine, Chosun University, 588,

Seosuk-dong, Dong-gu, Gwangju-city 501-717, Republic of Korea

Tel: +82-62-220-3126, Fax: +82-62-227-4575

E-mail: ns64902@hanmail.net/chosunns@chosun.ac.kr
}

*This study was supported by research funds from chosun university 2013 epidemiology, clinical presentation, surgical outcome and complications with a relevant literature review.

\section{MATERIALS AND METHODS}

Between 2005 and 2012, total 9 cases of lumbar discal cyst with symptomatic radiculopathy were selected for microscopic excision of discal cyst. The results were analyzed case by case. Patients (eight men and one woman) who ranged from 17 years to 37 in their age (mean age 27.1) had lesions which were varied from L2-L3 to L5-S1 level. All patients met the following criteria: (1) unilateral radicular leg pain, (2) discal cyst without disc herniation, spinal stenosis or instability, (3) no history of previous lumbar surgery. All patients had single level involvement and had radicular pain and numbness. Instrumentation wasn't performed in any patient. Specimens were sent in 7 patients for histologic study. In one patient, additional discectomy was performed due to disc bullging. All patients have been studied over at least 6 months and mean follow-up period was 9 months. At final follow-up, symptomatic improvement was evaluated by the visual analogue pain 
Table 1. Demographics of the patients with discal cyst

\begin{tabular}{|c|c|c|c|c|c|c|c|}
\hline Case & Age/sex & Level & Direction & Preop VAS & Postop VAS & MacNab's criteria & Complication \\
\hline 1 & $31 / M$ & L2-3 & Left & 8 & 2 & Excellent & - \\
\hline 2 & $20 / M$ & L4-5 & Right & 8 & 2 & Excellent & - \\
\hline 3 & $35 / M$ & L4-5 & Right & 7 & 2 & Excellent & - \\
\hline 4 & $17 / F$ & L5-SI & Left & 8 & 3 & Good & - \\
\hline 5 & $23 / M$ & L4-5 & Right & 8 & 3 & Good & - \\
\hline 6 & $27 / M$ & L4-5 & Right & 9 & 3 & Excellent & Dura tear \\
\hline 7 & $37 / M$ & L3-4 & Left & 6 & 4 & Good & - \\
\hline 8 & 29/M & $\lfloor 4-5$ & Left & 8 & 2 & Excellent & - \\
\hline 9 & $25 / M$ & L4-5 & Right & 8 & 2 & Excellent & - \\
\hline
\end{tabular}

VAS: Visual Analogue Scale
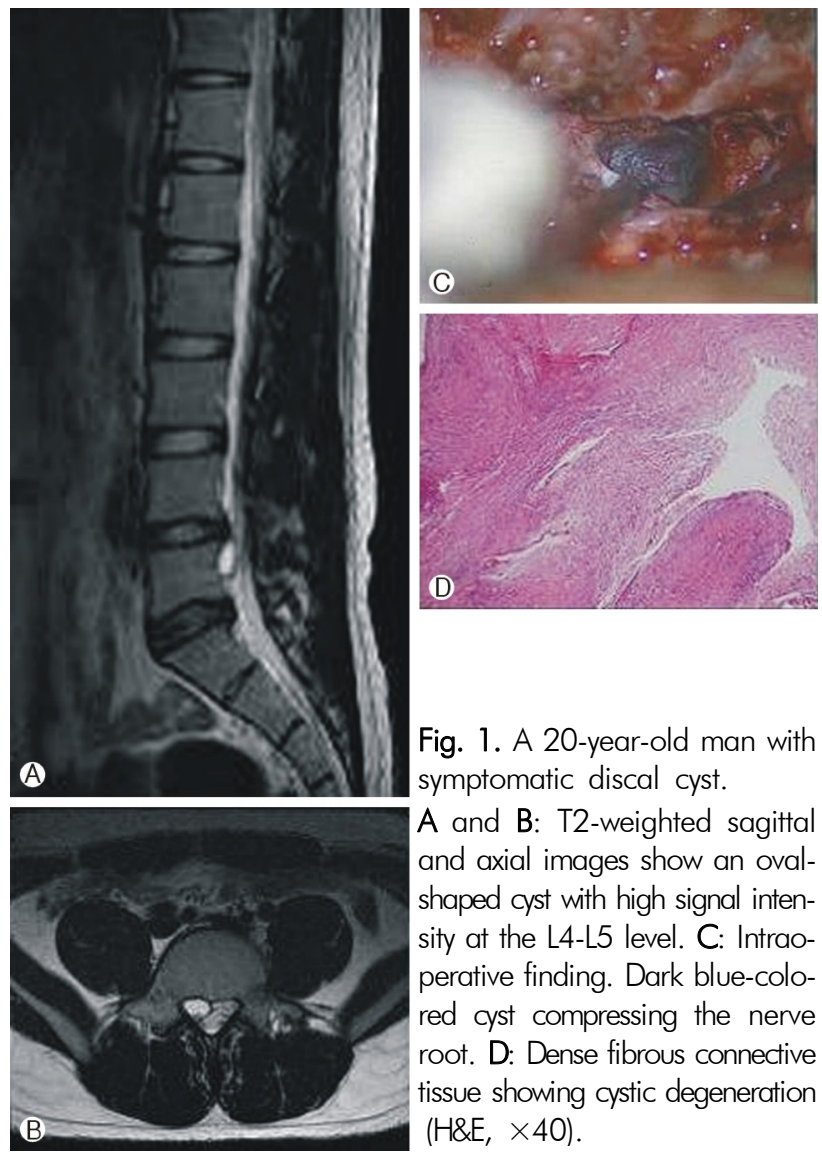

Fig. 1. A 20-year-old man with symptomatic discal cyst.

A and B: T2-weighted sagittal and axial images show an ovalshaped cyst with high signal intensity at the L4-L5 level. C: Intraoperative finding. Dark blue-colored cyst compressing the nerve root. D: Dense fibrous connective tissue showing cystic degeneration $(\mathrm{H} \& \mathrm{E}, \times 40)$.

score (VAS), and modified MacNab's criteria.

\section{RESULTS}

All of the patients suffered from unilateral lower extremity pain and positive tension sign. Although the straight leg raising test was positive in all patients, their low-back pain was mild. The preoperative magnetic resonance image (MRI) and
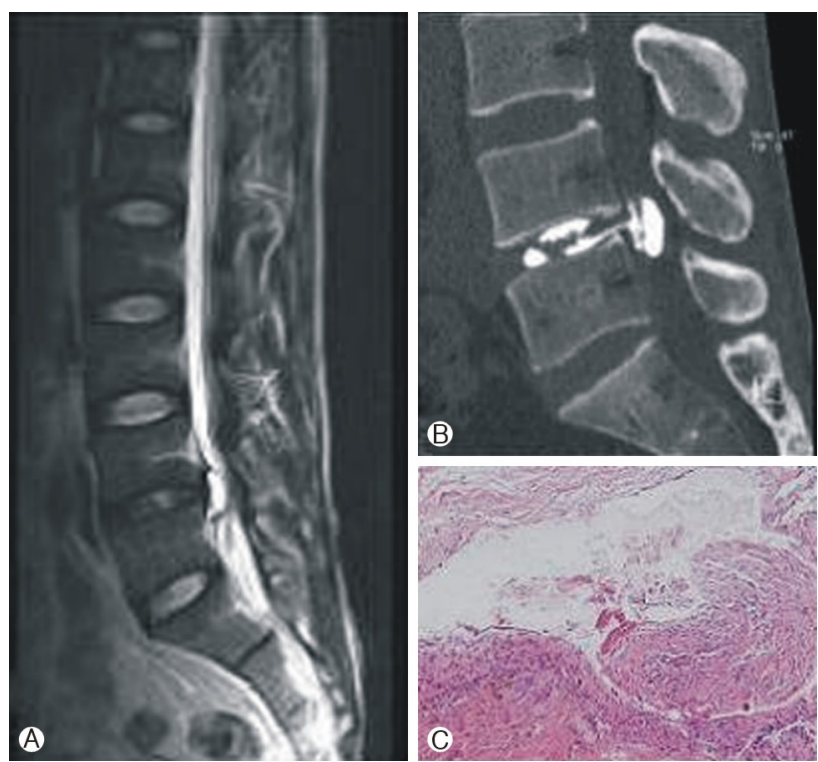

Fig. 2. A 35-year-old man with symptomatic discal cyst.

$\mathrm{A}$ and $\mathrm{B}$ : T2-weighted sagittal magnetic resonance image and computed tomographic scan after discography reveal the cyst connected to L4-L5 intervertebral disc. C: The cyst consisted of dense fibrocollagenous connective tissue with hemosiderin-laden macrophages.

3 dimensional-computed tomography (CT) with discogram in all cases showed the cyst connected to the intervertebral disc. Mean follow-up period was 9.6 months. All MR images of patients have shown the low signal intensity in T1-weighted images and high signal intensity in T2-weighted images, round to oval mass lesion on MRI, are compatible with a liquid-containing cyst. Numbness and radiating pain were improved in all patients at the end of evaluation period. The mean preoperative VAS score was 7.8 when compared with 2.6 in postoperative assessment (Table 1 ).

All patients obtained excellent or good results according to modified MacNab's criteria (excellent: 6, good: 3). Dural 


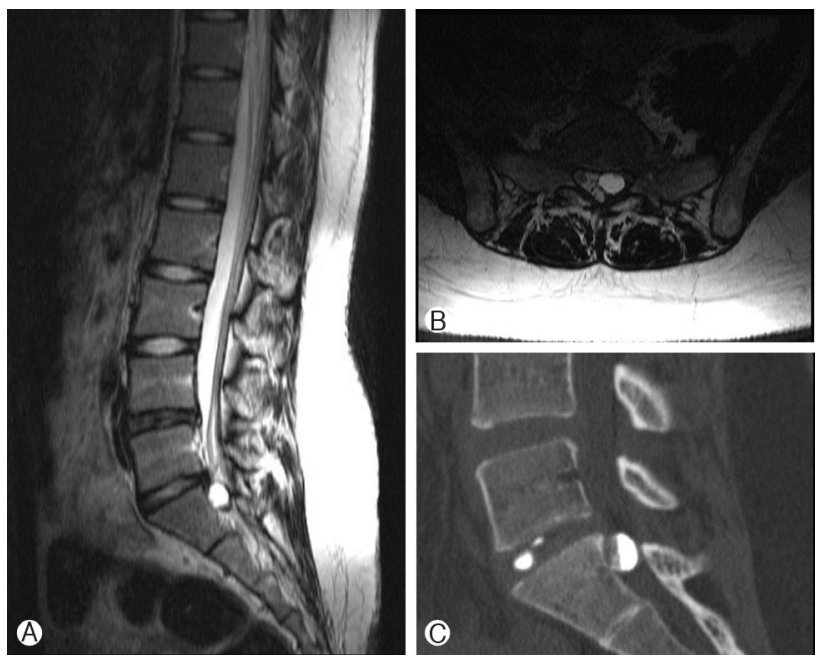

Fig. 3. A 17-year-old woman with symptomatic discal cyst. $A$ and $B$ : T2-weighted sagittal and axial magnetic resonance images reveal the discal cyst. C: Computed tomographic scan after discography confirms the cyst connected to L5-S1 intervertebral disc.

tear was noticed in one patient as an attempt was made to dissect the adhesion with the dura. Dural repair by artificial dural graft was performed, and fibrin glue was injected. There was no sign of persistent cerebrospinal fluid leakage and meningocele. There was no evidence of the recurrence of cyst during the follow-up period. Histological examination of the cysts in 7 patients revealed fibrous connective tissue without evidence of epithelial lining cell or disc materials (Fig. 1, 2 ,3).

\section{DISCUSSION}

A variety of cystic lesions may develop within the spinal canal, including perineural cysts, extradural arachnoid cysts, and synovial cysts of the facet joint, as well as an extruded disc fragment in the epidural space ${ }^{7,8}$. They are also a recognized cause of low back pain and radiculopathy. However, intraspinal extradural cysts that communicate with the intervertebral disc, referred to as "discal cysts," are very rare and difficult to distinguish from other kinds of lesions ${ }^{3,5)}$. However, the pathogenesis of discal cysts remains still unclear and several causes of these cysts have been postulated. Chiba et al. ${ }^{1)}$ hypothesized that an epidural hematoma from an underlying intervertebral disc injuries initially formed from hemorrhage of the epidural venous plexus that occurs in the space between the peridural membrane and the vertebral body, and discal cysts form most likely as a consequence of impairment of hematoma resorption. Kono et al. ${ }^{6}$ also insisted that the pathogenesis of discal cysts is focal degeneration of the inter- vertebral disc with fluid production similar to that of meniscal cysts of the knee and synovial cysts of the facet joint. The extruded fluid from the herniated disc material provokes an inflammatory response leading to reactive pseudomembrane formation and the development of a discal cyst. Whatever the pathogenesis may be, the intraspinal cyst itself, and not the intervertebral disc is responsible for the development of the symptoms. The clinical symptoms and signs of patients with discal cysts are similar to those of patients with a typical disc herniation manifesting as a unilateral radiculopathy. Myelographic or CT-myelographic findings of the cyst were similar to those for sequestrated lumbar disc herniation. However, neuroimaging investigations such as MR imaging, can be used to very accurately identify discal cysts. Discal cysts usually show low signal intensity on T1-weighted images and a high signal intensity on T2-weighted images. However, this signal can vary relying on the contents of fluid. The surrounding peripheral rim and the contents of the cyst are enhanced by Gd-DTPA on the enhanced MR images. A connecting channel between the cyst and the corresponding disc is the key to differentiate discal cysts from lumbar disc herniation. A connecting channel which serves as further diagnostic evidence of a discal cyst, can be confirmed on CT discography. Despite its invasive nature, it is possible to differentiate discal cysts from lumbar disc herniation or other intraspinal cysts. Pathologically, a discal cyst consists of a fibrous tissue capsule and some myxoid degeneration. Hemosiderin, inflammatory cells, or macrophages may also be seen in the histologic specimens. However, there is no evidence of disc material. The exact natural history of discal cysts remains unknown. There is only one case report of spontaneous regression without specific treatment ${ }^{2}$. Thus, a therapeutic strategy for discal cysts remains to be established. However, we believe that the operative indications for discal cyst are likely to be similar to those of lumbar disc herniation. In our study, discal cysts can be treated effectively by microscopic removal of the cyst with preservation of the disc, even though it is generally believed that a discal cyst may recur when the cyst is only partially removed. To provide a more definitive answer to the question of natural course and standard treatment for discal cysts, more cases of discal cyst accompanied by careful analysis and longterm follow-up are necessary.

\section{CONCLUSION}

Although a rare disease, the possibility of a discal cyst should be kept in mind as it may manifest with symptoms and signs resembling those of lumbar disc herniation. Microscopic exci- 
sion of the cyst without additional discectomy can be the standard treatment of discal cysts.

\section{REFERENCES}

1. Chiba K, Toyama Y, Matsumoto M, Maruiwa H, Watanave M, Nishiza-wa T: Intraspinal cyst communicating with the intervertebral disc in the lumbar spine: discal cyst. Spine (Phila Pa 1976) 26:2112-2118, 2001

2. Coscia MF, Broshears JR: Lumbar spine intracanalicular discal cysts : two case reports. J Spine Disord Tech 15:431-435, 2002

3. Lee HK, Lee DH, Choi CG, Kim SJ, Suh DC, Kahng SK, et al.: Discal cyst of the lumbar spine: MR imaging features. Clin Ima- ging 30:326-330, 2006

4. Lim HG, Kim HS, Kim SW, Shin H: Discal cyst diagnosed by radiologic finding. J Korean Neurosug Soc 41:418-420, 2007

5. Kim SH, Ahn SS, Choi GH, Kim DH: Discal cyst of the lumbar spine: a case report. Korean J Spine 9(2):114-117, 2012

6. Kono K, Nakamura H, Inoue Y, Okamura T, Shakudo M, Yamada R: Intraspinal extradural cysts communicating with adjacent herniated disks: imaging characteristics and possible pathogenesis. AJNR Am J Neuroradiol 20:1373-1377, 1999

7. Ogawa Y, Kumano K, Hirabayashi S, Aota Y: A ganglion cyst in the lumbar spinal canal. A case report. Spine 17:1429-1431, 1992

8. Onofrio BM, Mih AD: Synovial cysts of the spine. Neurosurgery 22:642-647, 1988 